\title{
Application d'une méthode immunoenzymatique à la sélection pour la stabilité de la stérilité mâle cytoplasmique chez la féverole (Vicia faba L)
}

\author{
N Berthaut 1, G Duc 1, P Dulieu 1, E Boudon-Padieu 2, H Dulieu 1 \\ ${ }^{1}$ INRA, station de génétique et d'amélioration des plantes, BV 1540, 21034 Dijon Cédex; \\ 2 INRA, station de recherches sur les mycoplasmes, BV 1540, 21034 Dijon, Cédex, France
}

(Reçu 26 juin 1990; accepté 29 november1990)

\begin{abstract}
Résumé - La stérilité mâle nucléocytoplasmique « 447 » de la féverole (Vicia faba L) manifeste de fréquentes instabilités qui s'expriment par des retours à la fertilité parmi les lignées en cours de conversion vers la stérilité. La mise en évidence de particules cytoplasmiques pseudovirales liées à la stérilité et la forte relation entre teneur en particules et degré de stabilité du maintien a permis d'envisager l'utilisation de cette teneur comme critère de sélection. Un test immunoenzymatique du type Enzyme linked immunosorbant assay (ELISA) après l'obtention d'un antisérum et d'immunoglobulines de classe $\mathrm{G}$ (lgG) a été développé pour mesurer la teneur en particules pseudovirales dans la plante. L'étude de cette teneur dans les divers organes de la plante, de leur répartition dans les différents niveaux foliaires, selon l'âge de la plante et selon la température, débouche sur une nouvelle méthode d'évaluation de la stérilité mâle nucléocytoplasmique «447». Cette méthode d'évaluation de la stérilité mâle est comparée à la méthode directe basée sur l'observation pollinique. La bonne héritabilité du caractère teneur en particules permet de sélectionner efficacement pour le maintien de la stérilité «447».
\end{abstract}

Vicia faba $\mathrm{L}$ = féverole / stérilité mâle cytoplasmique / particule pseudovirale / ELISA

Summary - Use of an immunoenzymatic assay in breeding for higher stability in cytoplasmic male sterile faba bean (Vicia faba L). The nucieocytoplasmic male sterility "447" of faba bean Nicia faba $L$ ) shows frequent instabilities expressed by the occurrence of male fertile plants in backcross progenies. The discovery of cytoplasmic viruslike particles (VLPS) linked to male sterility and the observation of a strong relationship between particle contents and stability level led the breeder to utilise virus-like particle content as a selection criterion. An enzyme linked immunosorbant assay (ELISA) test has been developed through antiserum and IgG production for quantifying the VLPS. The evaluation of VLP contents in different plant organs (tables I and II), their repartition according to node level (fig 2), plant age (tables III and IV) and temperature have led to a new method for male sterility evaluation in breeding programs. The efficiency of this method appears higher when compared to the direct method of pollen observation (fig 5). The good heritability of VLP content character analyzed in figs 3 and 4 allows efficient selection for stabilizing male sterility «447».

Vicia faba $L$ = faba bean / cytoplasmic male sterility / virus-like particle / ELISA

\section{INTRODUCTION}

La stérilité mâle nucléocytoplasmique «447» de la féverole manifeste de fréquentes instabilités qui s'expriment par des retours à la fertilité parmi les lignées en cours de conversion vers la stérilité (Bond et al, 1986; Thiellement, 1977a; Duc, 1978; Picard et al, 1982).

L'évaluation du degré de stérilité d'une plante par l'examen de son pollen permet une sélection de lignées mainteneuses (Duc, 1978). Cepen- dant cette méthode a un pouvoir de détection limité qui conduit à retenir beaucoup de matériel. Une bonne partie de celui-ci s'avère médiocrement mainteneur, même après de nombreux backcross (BC).

Edwardson et al (1976) ont mis en évidence des particules cytoplasmiques pseudovirales (VLPs $=$ virus like particles) dans les cellules des plantes exprimant la stérilité mâle " 447 " de Vicia faba et leur disparition avec les retours à la fertilité. La liaison entre teneur en particules et degré 
de stabilité du maintien mesurée par Duc et al (1984), a permis d'envisager l'utilisation de la teneur en VLPS comme critère de sélection.

La purification jusqu'à homogénéité physique, la préparation d'un antisérum et d'lgG ont permis la détection des VLPS par ELISA (Dulieu et al, 1988).

Pour l'adaptation de ce test à la sélection, il était nécessaire dans un premier temps, d'étudier l'amplitude décelable et la répartition des VLPs, et ensuite de définir un protocole précis d'échantillonnage pour s'assurer de la fidélité et $\mathrm{du}$ pouvoir discriminant $\mathrm{du}$ test. Dans un deuxième temps, nous avons étudié la corrélation entre les résultats obtenus en ELISA et ceux obtenus avec les précédentes méthodes d'évaIuation de la stérilité mâle.

\section{MATÉRIEL ET MÉTHODES}

La lignée $\mathrm{Ad} 23$, issue d'une sélection effectuée au cours de 12 générations de back cross (Berthelem, INRA Rennes) possède une très bonne aptitude au maintien de la stérilité mâle «447». La version mâlestérile de cette lignée sert de témoin de référence.

Afin de pouvoir disposer d'une collection de lignées mâle-stériles à potentialités différentes, une population a été constituée à partir d'un ensemble de 25 lignées mainteneuses, intercroisées en fécondation libre pendant 3 générations pour assurer un brassage génétique suffisant. De là ont été choisies, pour leurs qualités agronomiques, 100 plantes dites «GF». Elles ont été hybridées avec la lignée Ad23 mâle-stérile et leurs descendants ont servi de parents mâles récurrents jusqu'au $\mathrm{BC}_{4}$.

Les plantes sont cultivées en serre hors gel ou en champ, suivant la saison. Dans certaines expériences particulières, elles sont placées en chambres climatisées (température constante, $20^{\circ} \mathrm{C}$ ou $25^{\circ} \mathrm{C}, 16 \mathrm{~h}$ d'éclairage, $\left.130 \mathrm{~W} / \mathrm{m}^{2}\right)$.

La teneur en VLPs est estimée avec un ELISA de type sandwich utilisant des anticorps polyclonaux antiVLPs (Dulieu et al, 1988).

L'extraction des VLPS est réalisée à partir de disques foliaires de $10 \mathrm{~mm}$ de diamètre au stade feuille étalée, de pistils entiers au stade ouverture de la fleur ou encore à partir de graines en formation, extraites de jeunes gousses de $2 \mathrm{~cm}$ de longueur.

Les échantillons sont broyés (dans un tube Eppendorf) avec 5 volumes de tampon d'extraction (saccharose 0,4 mol. $\mathrm{I}^{-1}$, glycérol $10 \%, \mathrm{KCl} 10 \mathrm{mmol}^{-1} \mathrm{MgCl}_{2}$ 5 mmol. $\left.\right|^{-1}$, DDT $2 \mathrm{mmol} . \mathrm{I}^{-1}$, TRIS $50 \mathrm{mmol} . \mathrm{I}^{-1}, \mathrm{pH}$ $7,5)$ puis centrifugés à $18000 \mathrm{~g}$ durant $30 \mathrm{~min}$ à $4^{\circ} \mathrm{C}$.

Le surnageant est dilué 45 fois. Cent microlitres sont incubés dans les puits de plaques de microtitration (Nunc) revêtues par les lgG-anti-VLPs $\left(A C_{1}\right)$, préparés selon la méthode de Dulieu et al (1988) à raison de 4 puits par extrait. Après incubation, les puits sont lavés avec un tampon caséine afin d'éliminer les antigènes fixés non spécifiquement. Les puits sont ensuite incubés en présence d'anticorps anti-VLPs, conjugués à la phosphatase alcaline $\left(\mathrm{AC}_{2}\right)$ par la méthode d'Avrameas (1969).

Après lavage des puits, l'activité enzymatique fixée est mesurée par incubation $30 \mathrm{~min}$ à $20^{\circ} \mathrm{C}$ avec le substrat PNPP (para-nitro-phényl-phosphate $1 \mathrm{mg} / \mathrm{ml}$ de tampon diéthanol amine). La coloration due à la formation de para-nitro-phénol est mesurée en densité optique (DO) à $405 \mathrm{~nm}$. Cette méthode est dérivée de Boudon-Padieu (1989).

Au cours de l'analyse des résultats, les valeurs de DO sont utilisées telles quelles ou en \% de celle du témoin. Afin de normaliser la distribution des données, les valeurs transformées $1 / x$, si $x=D O$ (échantillons) / DO $(\operatorname{ad} 23 \times 100)$, sont utilisées pour l'analyse de variance dans les cas où l'ensemble des pourcentages se situent aux environs de 100 (transformation préconisée par Steel et Torrie, 1960). Les comparaisons de moyennes ont été réalisées selon le test de Newman et Keuls (1981).

L'évaluation de la stérilité du pollen s'effectue au microscope sur 300 grains de pollen cólorés selon Alexander (1969) par le pourcentage de grains contenant du cytoplasme.

Une observation visuelle de la fertilité pollinique de 2 fleurs par plante permet de détecter le nombre de plantes ayant effectué un retour complet à la fertilité.

\section{RÉSULTATS}

\section{Recherche de dilutions optimales des anticorps et antigènes}

Les dilutions optimales des $A C_{1}$ et $A C_{2}$ ont été déterminées à $1 / 1000$ et $1 / 5000$, respectivement, de manière à obtenir une variation linéaire de $\mathrm{DO}$ en fonction du logarithme de la dilution de l'extrait foliaire d'Ad23.

La dilution de l'extrait foliaire a été fixée à $1 / 45$; elle correspond au milieu de la zone de réponse linéaire (1/16 à 1/256) observée sur l'étendue des dilutions testées : 1/2 à 1/512 (fig 1). Dans cette zone, le test de linéarité donne une corrélation $r=0,98$ (38 ddl) significative au seuil de $1 \%$.

\section{Recherche des VLPs dans différents organes}

Dans un premier temps, la recherche de la teneur en VLPS a été effectuée dans les pistils et dans les feuilles, simultanément sur 5 feuilles et 


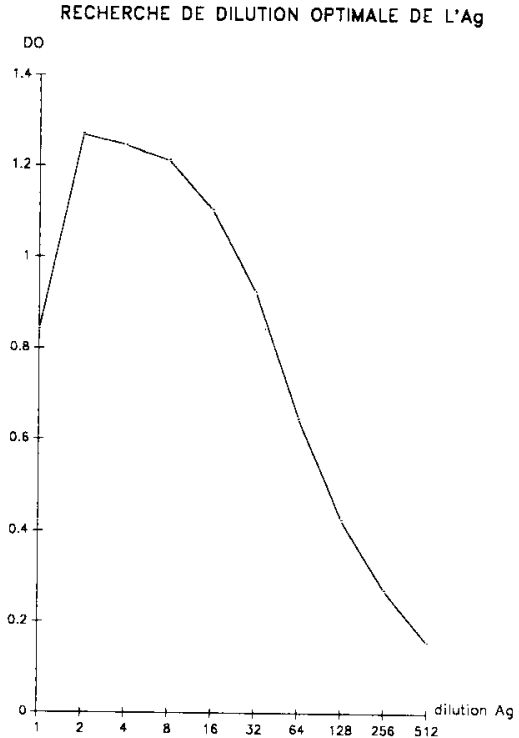

Fig 1. Influence de la dilution de l'Ag sur la réponse ELISA. Chaque point représente la moyenne de 8 répétitions de la même dilution.

3 pistils pour chacune des 6 plântes Ad23 mâlestériles utilisées. Dans un deuxième temps, elle a été réalisée sur des jeunes graines prélevées $15 \mathrm{j}$ après pollinisation des plantes mâle-stériles Ad23 par leur mainteneur. Les croisements sont réalisés sur 5 plantes et 3 fleurs par plante (les phénomènes d'avortement des fleurs, fréquents chez la féverole, n'ont pas permis de disposer dans tous les cas des 3 jeunes gousses par plante).

Les résultats sont reportés dans les tableaux I et II; ils sont exprimés en somme de DO pour 4 répétitions du même extrait. Ces tableaux montrent l'existence d'une variabilité entreplantes et entre organes, de la teneur en VLPs.

Tableau I. Mesure ELISA (exprimée en $\Sigma D O$ ) des teneurs en VLPs de feuilles et pistils chez 6 plantes de la lignée Ad23.

\begin{tabular}{lll}
\hline Noplantes & $\Sigma D O$ feulles & $\Sigma D O$ pistils \\
\hline 1 & & \\
2 & 0,70 & 1,11 \\
3 & 2,07 & 2,21 \\
6 & 1,75 & 2,30 \\
7 & 1,78 & 1,98 \\
8 & 2,39 & 2,40 \\
m & 2,66 & 2,69 \\
mainteneur & 1,89 & 2,11 \\
CV (\%) & 0,019 & - \\
résiduel & 32,8 & 23,6
\end{tabular}

résiduel

Coefficient de corrélation entre les moyennes : 0,96
Malgré une tendance à la supériorité de la concentration des VLPs dans les pistils (différence non significative) par rapport aux feuilles de la même plante, nous avons choisi de réaliser l'estimation de la teneur en particules sur feuilles, en vue d'opérer une sélection sur le degré de stérilité au stade le plus précoce possible.

L'estimation de la teneur en VLPs de jeunes graines ne présente pas d'intérêt en tant que critère de sélection, puisqu'elle est destructrice. Elle a été réalisée dans le seul but de connaître l'étendue de la répartition des VLPS dans la plante et montre que les jeunes graines qui transmettent la stérilité présentent une variabilité intergraine, et une réaction positive bien inférieures à celles des feuilles.

On peut noter que la teneur faible mesurée sur graine reflète davantage celle des cotylédons que celle des tissus méristématiques, compte tenu de la masse très supérieure des premiers.

\section{Mesure des VLPs dans les feuilles}

Ayant choisi les feuilles pour nos mesures, il convient ensuite d'en définir le mode de prélèvement.

\section{Recherche d'un stade précoce}

Deux séries de mesures ont été étudiées sur un ensemble de plantes élevées en chambre climatisée.

Dans la série 1 , les prélèvements sont réalisés sur 15 plantes de la lignée Ad23 selon 3 modalités :

- mesure de la teneur sur la 1re feuille dès son émergence (prélèvement 1);

- mesure sur la même feuille 15 j plus tard (prélèvement 2);

- mesure de la teneur au stade floral sur un échantillon moyen prélevé sur 5 feuilles des nœuds foliaires consécutifs 1-5 (prélèvement 3).

Les résultats se trouvent analysés dans le tableau III. L'analyse de variance indique un seuil de signification supérieur à $1 \%$ pour les effets plantes, prélèvement, et pour l'interaction plante $x$ prélèvement. II ressort un carré moyen prépondérant pour le facteur prélèvement, largement expliqué par une faible DO dans la modalité 1 . 
Tableau II. Mesure ELISA (exprimée en LDO) des teneurs de VLPs de jeunes graines de la lignée Ad23.

\begin{tabular}{llll}
\hline No plantes & Nofleurs & Nb graines & $\Sigma D O$ \\
\hline 1 & 1 & 3 & 0,58 \\
1 & 2 & 2 & 0,48 \\
1 & 3 & 4 & 0,67 \\
3 & 1 & 1 & 0,52 \\
3 & 2 & 2 & 0,41 \\
7 & 1 & 2 & 0,66 \\
7 & 2 & 2 & 0,69 \\
9 & 1 & 2 & 0,64 \\
11 & 1 & 2 & 0,55 \\
11 & 2 & 3 & 0,47 \\
11 & 3 & 3 & 0,50 \\
m & & & 0,56 \\
mainteneur & 1 & 1 & 0,03 \\
CV (\%) & & & \\
résiduel : 15,8 & & &
\end{tabular}

Tableau III. A. Analyse de variance des mesures ELISA (unités de DO) sur feuilles, réalisées selon 3 modalités de prélèvement, sur différentes plantes de la lignée Ad23. B. Classement des moyennes du facteur prélèvement. (test de Newman et Keuls, seuil de 5\%).

\section{A}

Analyse de variance

Origine de

la variation

ddl Carré TestF

Seuil de

moyen signification

Totale
Facteur
- prélèvement
- plante
- Interaction
prél x pl

0,0088

Résiduelle

CV $=12,9 \%$

$\begin{array}{lll}2 & 0,18 & 208 \\ 14 & 0,0507 & \\ 28 & 0,00653 \\ 315 & 0,00086\end{array}$

$\begin{array}{ll}208 & 1 \% \\ 58 & 1 \% \\ 75 & 1 \%\end{array}$

B

$\begin{array}{lrr}3 & 0,25 & \mathrm{a} \\ 2 & 0,25 & \mathrm{a} \\ 1 & 0,18 & \mathrm{~b}\end{array}$

Dans la série 2, les prélèvements sont réalisés sur 36 plantes appartenant à 6 familles de la population GF en $\mathrm{BC}_{4}$ selon 2 modalités :

- mesure sur la 1 re feuille dès son émergence (prélèvement 1);
- mesure au stade floral sur un échantillon prélevé sur 5 feuilles des nœuds consécutifs 1-5 (prélèvement 2).

Les résultats de DO exprimés en \% du témoin Ad23 se trouvent analysés dans le tableau IV. 
Tableau IV. A. Analyse de variance des mesures ELISA sur feuille (\% du témoin Ad23), réalisées selon deux modalités de prélèvement, sur 6 familles GF de backcross 4. B. Classement des moyennes (test de Newman et Keuls seuil $5 \%)$.

A

Analyse de variance

Origine de

la variation

$d d l$

Carré

Test F

Seuil de

moyen

signification

\begin{tabular}{|c|c|c|c|}
\hline Totale & 71 & 1506 & \\
\hline $\begin{array}{l}\text { Facteur famille } \\
\text { Facteur prélèvement } \\
\text { Interac famille } x \text { prél }\end{array}$ & $\begin{array}{l}5 \\
1 \\
5\end{array}$ & $\begin{array}{r}14009 \\
3376 \\
1031\end{array}$ & $\begin{array}{r}29,6 \\
7,1 \\
2,2\end{array}$ \\
\hline $\begin{array}{l}\text { Résiduelle } \\
C V=24,7 \%\end{array}$ & 60 & 473 & \\
\hline
\end{tabular}

CV $=24,7 \%$

B

\begin{tabular}{llll}
\hline \multirow{2}{*}{ Facteur } & Numéro & Moyenne & Groupes homogènes \\
\hline \multirow{2}{*}{ Famille } & 411 & & \\
& 201 & 153,0 & $\mathrm{a}$ \\
& 214 & 83,8 & $\mathrm{~b}$ \\
& 591 & 83,6 & $\mathrm{~b}$ \\
& 281 & 82,6 & $\mathrm{~b}$ \\
Prélèvement & 872 & 72,6 & $\mathrm{~b}$ \\
& 2 & 51,8 & $\mathrm{c}$ \\
& 1 & 94,8 & $\mathrm{a}$ \\
\hline
\end{tabular}

De façon conforme au résultat de l'expérience précédente, le prélèvement, à la fois plus tardif et couvrant plus de nœuds foliaires donne une réponse supérieure en ELISA. Cette expérience montre aussi que l'appréciation du degré de stérilité de la population "GF» diffère significativement entre les 2 prélèvements. L'analyse individuelle des 2 prélèvements 1 et 2 aboutit à des coefficients de variation respectivement de 35 et $12 \%$, et à des valeurs de $F$ du facteur famille respectivement de 8 et 57 . On en déduit une meilleure précision et un meilleur pouvoir discriminant pour un prélèvement de modalité 2 .

\section{Influence du niveau foliaire}

Pour une meilleure connaissance d'un prélèvement qui regroupe plusieurs nœuds foliaires à la base de la plante, nous avons, un jour donné, prélevé individuellement les nœuds foliaires 3,4 ,
6,7 , et 9 sur 2 plantes de la lignée Ad23 (la moyenne des mesures sur 2 folioles par feuille a été considérée comme la valeur de cette feuille). La figure 2 illustre les résultats obtenus. L'analyse de variance ne permet pas de détecter d'effet significatif pour le niveau foliaire et les

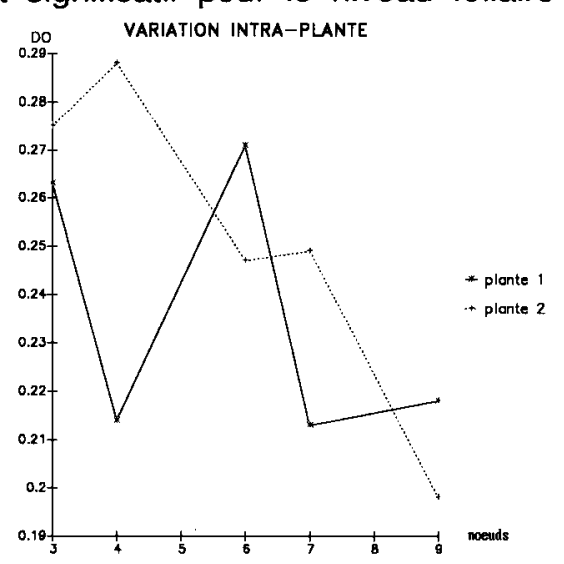

Fig 2. Influence du niveau foliaire sur la valeur ELISA chez 2 plantes de la lignée mâle-stérile Ad23. Chaque point représente la moyenne des sommes de DO de 2 folioles par feuille. 
feuilles d'une même orthostique tandis que l'interaction plante $\mathrm{x}$ niveau foliaire est significative à $1 \%$. Ce résultat suggère que les variations intraplante des contenus en particules ne sont pas déterminées par la morphogenèse de la plante mais seraient aléatoires et/ou sous l'influence des facteurs du milieu.

\section{Influence du milieu}

L'influence de l'environnement sur la stabilité de la stérilité mâle cytoplasmique chez la féverole a été démontrée par plusieurs auteurs (Duc, 1978; Letouzey, 1981). En fonction de sa liaison étroite avec la teneur en particules, il était important d'étudier l'effet de la température sur la valeur ELISA et notamment sur la lignée Ad23 utilisée comme témoin de référence; ceci dans des conditions de milieu moyennes pour la culture de la féverole et non inductrices de retour à la fertilité chez Ad23.

L'étude a porté sur 2 lots de la lignée Ad23, constitués de 15 plantes chacun, élevés en chambre de culture avec $16 \mathrm{~h}$ d'éclairement, l'un à $20^{\circ} \mathrm{C}$, l'autre à $25^{\circ} \mathrm{C}$. Le test ELISA a été réalisé sur un prélèvement de 5 étages foliaires par plante. Les résultats exprimés en somme de DO sont étudiés à raison de 4 répétitions par prélèvement. Les moyennes de DO sont respectivement de 1,05 à $25^{\circ} \mathrm{C}$ et de 0,50 à $20^{\circ} \mathrm{C}$, déclarées significativement différentes au seuil $5 \%$ par un test $t$. Cependant, on enregistre des coefficients de variation interplantes et intratraitement beaucoup plus élevés à $20^{\circ} \mathrm{C}(42 \%)$ qu'à $25^{\circ} \mathrm{C}(13 \%)$.

\section{Essai de conservation des VLPs}

Pour rendre le critère «teneur en VLPS" plus opérationnel dans le cadre d'un programme de sélection, il peut être utile de développer une technique de conservation des échantillons qui permette d'en différer les dosages. Un témoin utilisé par une technique de conservation serait également un excellent témoin de calibrage. Pour cet objectif, nous avons évalué l'effet de la congélation à $-80^{\circ} \mathrm{C}$.

Deux types d'essais ont été réalisés :

- le premier a porté sur la congélation des échantillons de feuilles. Les résultats (tableau V) montrent que le procédé de conservation altère sensiblement les différences entre les génotypes conservés;

- le deuxième réalisé sur la congélation de surnageant obtenu lors de l'extraction des VLPs a montré une réduction de $30 \%$ des valeurs de DO après congélation et des inversions des classements entre plantes.

L'ensemble de ces essais de conservation n'aboutit pas à une méthode fiable et seuls, seront considérés pour la suite, les résultats sur matériel frais.

\section{Performance et précision du test immunoenzymatique en sélection}

Au regard des résultats précédents, nous avons pu juger de la variabilité interplantes mise en évidence par le test ELISA, aussi bien dans la lignée Ad23 (tableaux II et III) que dans la population GF (tableau IV).

Tableau V. Effet de la conservation de feuilles par congélation sur la mesure ELISA de teneur en VLPS (moyenne de DO).

\begin{tabular}{llllr}
\hline & $\begin{array}{l}\text { Frais } \\
\text { Plantes GF }\end{array}$ & \% Ad23 & DO & Congelé \\
& & & & \\
$31-1-2$ & 0,36 & 46 & 0,40 & 48 \\
$28-2-2$ & 0,57 & 73 & 0,46 & 43 \\
$29-2-1$ & 0,40 & 51 & 0,36 & 8 \\
$30-2-2$ & 0,08 & 10 & 0,07 & 58 \\
$30-2-1$ & 0,63 & 81 & 0,48 & \\
Moyenne & 0,41 & & 0,35 & \\
Ad23 (moyenne & & & & \\
de 2 plantes) & 0,78 & & & \\
\end{tabular}




\section{Étude de l'héritabilité du caractère teneur en particules}

Les teneurs en particules ont été mesurées dans la population $\mathrm{GF}$ à 3 générations du $\mathrm{BC}_{1}$ au $\mathrm{BC}_{3}$ pour certaines familles et pour 4 générations du $\mathrm{BC}_{1}$ au $\mathrm{BC}_{4}$ pour les autres. Les coefficients de corrélation calculés pour les valeurs $1 /(\%$ Ad23) étaient de : 0,73 entre $\mathrm{BC}_{1}$ et $\mathrm{BC}_{2}$ (significatif au seuil de $1 \%$; figure 3); 0,74 entre $\mathrm{BC}_{2}$ et $\mathrm{BC}_{3}$ (significatif au seuil de $1 \%$ ); 0,73 entre $\mathrm{BC}_{3}$ et $\mathrm{BC}_{4}$ (significatif au seuil de 1\%; fig 4) alors que les coefficients de corrélation correspondants, relatifs à la stérilité pollinique évaluée en microscopie optique avaient une valeur inférieure ou égale à 0,28 et étaient non significatifs.

Ces résultats montrent donc, pour le caractère «teneur en VLPs», une héritabilité très supérieure à ce qu'elle est pour le caractère «fertilité pollinique», ce qui suggère une sensibilité supérieure de ce dernier aux facteurs du milieu.

\section{Correspondance ELISA - Fertilité pollinique}

\section{Le test ELISA comparé au test pollinique} montre :

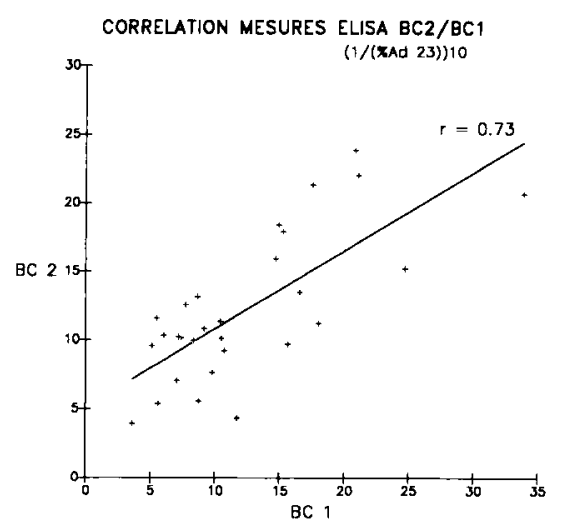

Fig 3. Liaison entre les générations de backcross 1 et 2 pour le caractère teneur en particules mesuré en ELISA. valeurs transformées $1 /(\%$ Ad23).

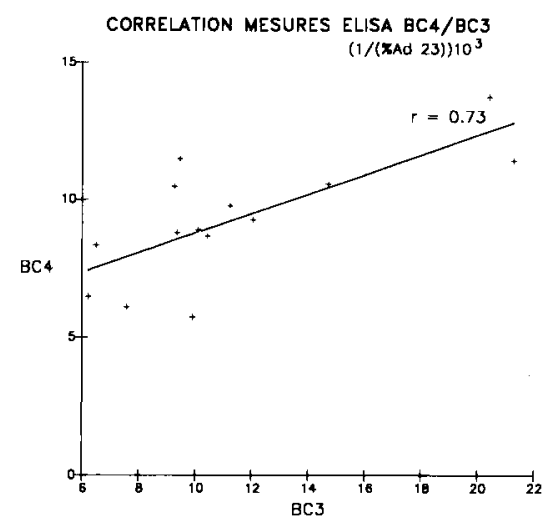

Fig 4. Liaison entre les générations de backcross 3 et 4 pour le caractère teneur en particules mesuré en ELISA. Valeurs transformées $1 /(\%$ Ad23).
- pour les valeurs $98-100 \%$ de grains de pollen fertiles, une réponse nulle en ELISA;

- pour les valeurs 1-97\% de grains de pollen fertiles, une réponse moyenne à faible en ELISA;

- pour la valeur $0 \%$ de grains de pollen fertiles ou contenant un peu de cytoplasme, qui correspond au degré de stérilité maximal mesurable en microscopie, le test ELISA exprime une variabilité : les 2 histogrammes de la figure 5 résument les résultats obtenus en valeur ELISA sur différentes lignées de GF à 2 générations pour la seule valeur $0 \%$ du test pollinique.

Ainsi, le test ELISA permet d'exploiter une variabilité préalablement non décelable.

\section{Étude de la liaison de la teneur en VLPs à l'observation visuelle du pollen}

L'observation visuelle du pollen, en raison de sa rapidité, permet l'examen d'un grand nombre d'individus en les rangeant en 2 catégories mâle-stériles ou mâle-fertiles. Nous avons estimé ainsi le nombre de plantes ayant réalisé un retour complet à la fertilité dans différentes descendances GF.
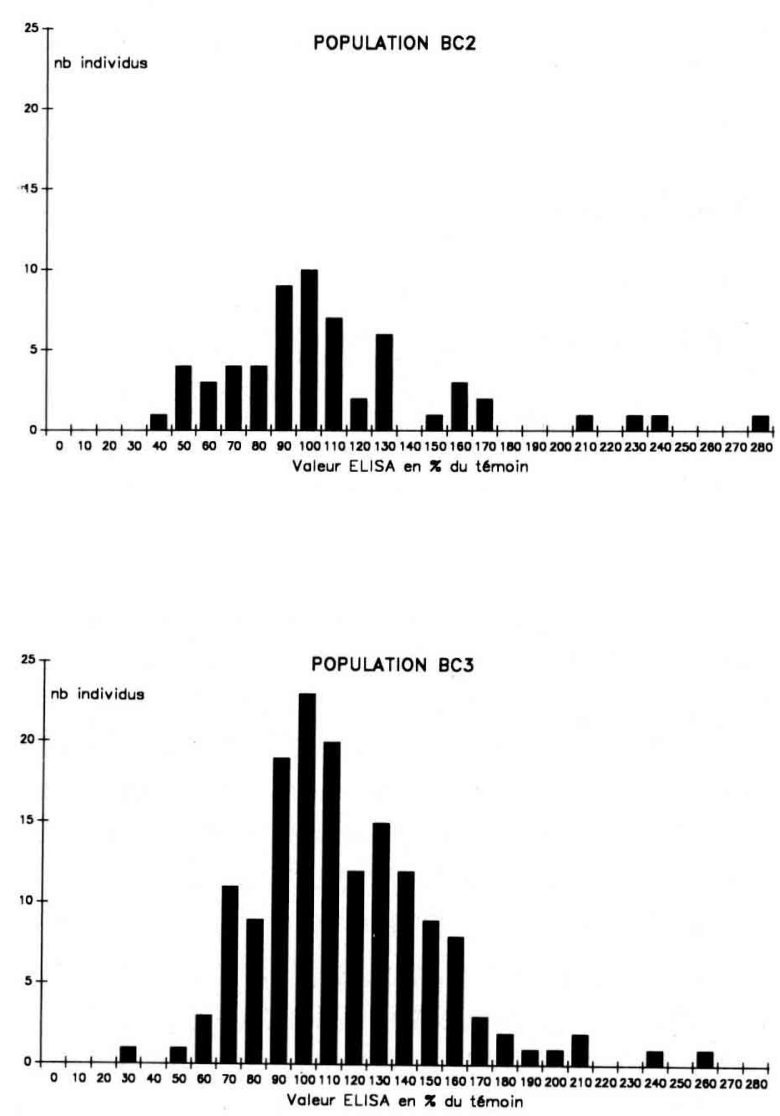

Fig 5. Étendue de la variation de la valeur ELISA (\% Ad23) dans 2 populations de génotypes présentant une valeur 0 de fertilité pollinique, aux $2^{\theta}$ et $3^{\ominus}$ générations de backcross. 
Tableau VI. Analyse de l'effet d'une sélection en backcross 3 simulée à 2 seuils de valeur ELISA (104 et 111\% Ad23) sur la fréquence de retours à la fertilité observée en backcross 4 .

\begin{tabular}{lllll}
\hline $\begin{array}{l}\text { Valeur moyenne ELISA (\% Ad23) } \\
\text { mesurée sur 5-9 plantes } \\
\text { mâle-stériles dans une descendance }\end{array}$ & $\begin{array}{l}\text { Nombre de } \\
\text { descendances }\end{array}$ & $\begin{array}{l}\text { Nombre de plantes de chaque } \\
\text { phénotype dans les descendances }\end{array}$ & $\chi^{2}$ \\
\cline { 2 - 4 } & & $\begin{array}{l}\text { Mâle-fertile } \\
\text { (réversions) }\end{array}$ & \\
\hline & 12 & 694 & $14(2,0 \%)$ & $P<0,01$ \\
$>104$ & 18 & 713 & $107(13,0 \%)$ & \\
$<104$ & 8 & 352 & $4(1,1 \%)$ & $P<0,01$ \\
$>111$ & 22 & 1055 & $117(10,0 \%)$ & \\
$<111$ & & & & \\
\hline
\end{tabular}

Le tableau VI donne pour une troisième génération de back cross, la répartition des plantes selon les critères ELISA et fertilité pollinique. En imposant un seuil de sélection, soit de $104 \%$ de la valeur d'Ad23, soit de $111 \%$ de cette valeur, on note par un test de $\chi^{2}$, significatif au seuil $1 \%$, une réduction des fréquences de réversion induite par chacun de ces seuils de sélection qui respectivement conduisent à 2,0 et $1,1 \%$ de plantes mâle-fertiles dans les descendances. $\mathrm{La}$ différence entre les 2 niveaux de sélection testée par un $\chi^{2}$ croisé est, quant à elle, non significative.

\section{DISCUSSION}

\section{Méthodologie}

L'appréciation par la méthode immunoenzymatique du degré de stérilité au stade jeune ne peut s'effectuer que sur un nombre restreint de feuilles. La distribution intraplantes des VLPS étant aléatoire, il est certain que le choix du seul stade précoce ne peut conduire qu'à une appréciation biaisée du degré de stérilité. Pour ces raisons, nous avons fixé le stade d'évaluation proche du début de la floraison, nous laissant la possibilité de prendre un échantillon moyen sur 5 feuilles, composé des nœuds consécutifs 1 à 5 , en-dessous du 1 er nœud floral.

Les variations de valeurs de DO enregistrées entre les expériences sur la lignée Ad23 qui sont partiellement expliquées par la sensibilité des VLPs à la température, posent le problème du témoin de référence pour l'estimation de la valeur des lignées. Nous avons résolu cette ques- tion par l'utilisation comme témoin de calibrage de plantes de la lignée Ad23, cultivées dans les mêmes conditions de milieu que les plantes étudiées.

D'après nos résultats, l'estimation de la teneur en VLPS d'une plante est une résultante de l'âge de cette plante et de la représentativité de l'échantillon prélevé. II faut bien sûr aussi tenir compte de la variabilité interplantes dans la lignée $\mathrm{Ad} 23$, déjà démontrée précédemment (Thiellement, 1977b). La somme de ces expériences et l'étude d'échantillonnage proposée par le logiciel STAT ITCF montrent que le témoin doit être constitué d'au moins 4 plantes de la lignée de référence et de même âge que les plantes à tester. De même, on peut considérer que la mesure sur une plante unique dans une descendance de backcross est insuffisante pour donner la valeur de cette descendance.

La congélation du matériel vivant ou du surnageant aurait permis de fixer le témoin pour une série d'expériences mais les fluctuations des teneurs en VLPs après congélation nous l'interdisent.

\section{Intérêt du test immunoenzymatique pour la sélection de lignées mâle-stériles}

La méthode d'évaluation de l'aptitude au maintien d'une plante par la stérilité de son pollen comporte des imperfections. D'une part, elle se veut prédictive du potentiel de transmission de la stérilité mâle par les ovules : cette corrélation mesurée dans différents travaux antérieurs (Duc, 1978; Thiellement, 1977c, 1982), bien que souvent significative, a une valeur assez moyenne. D'autre part, dans la méthode pollinique, l'éva- 
luation est basée sur la stérilité, plus ou moins importante, du pollen. Ce degré de stérilité est l'expression d'une plus au moins grande précocité de la dégénérescence du pollen (Moussel, 1987), qui n'est pas obligatoirement liée à un plus grand pouvoir de maintien de la stérilité. La notation de fertilité pour la méthode pollinique est limitée à la note 0 dont on sait qu'elle regroupe des lignées à niveaux de stabilité variables. II apparaît d'après notre expérience, que le critère teneur en VLPs soit plus proche de l'expression du potentiel du génotype que le critère de fertilité pollinique. On peut attribuer ceci, d'une part à une sensibilité différentielle des 2 critères à l'environnement et d'autre part, à la limite de sensibilité différentielle des 2 techniques.

Une forte héritabilité, un bon pouvoir discriminant et une bonne liaison avec le potentiel de stabilité des lignées sont les qualités du test immunoenzymatique de dosage de VLPs.

Lors du calcul de la corrélation entre les valeurs ELISA des $\mathrm{BC}_{3}$ et $\mathrm{BC}_{4}$ des lignées $\mathrm{GF}$, nous avons été amenés à retrancher 2 familles dont les valeurs particulières écartaient significativement la population d'une distribution normale. Elles étaient caractérisées par des valeurs ELISA, fortes en $\mathrm{BC}_{3}$ et très faibles en $B C_{4}$, et par ailleurs exprimaient de nombreux retours à la fertilité au $\mathrm{BC}_{4}$. On peut penser qu'il existe chez ces 2 familles, des gènes mineurs récessifs de restauration que le test ELISA n'avait pas détectés sous la forme hétérozygote du $B_{3}$. L'hypothèse de l'existence de tels gènes est soutenue par des résultats antérieurs d'analyses de croisement diallèle (Duc et Huglo, 1984).

L'étude de la teneur en VLPs, établie sur 3 générations, a pris en compte la mise au point du test immunoenzymatique, notamment les problèmes relatifs à l'échantillonnage et au témoin. Avec le protocole que nous avons choisi, nous obtenons une corrélation satisfaisante entre les différentes générations pour la teneur en VLPs et une bonne liaison de celles-ci à la stabilité de la stérilité mâle cytoplasmique. Ceci est tout à fait encourageant pour l'efficacité de la sélection de ce caractère et pour celle de lignées bonnes mainteneuses de stérilité.

\section{CONCLUSION}

Le test immunoenzymatique de type ELISA que nous avons mis au point, permet de sélectionner pour le pouvoir de maintien de lignées mâlestériles de féverole.
II demeure cependant que les gènes mineurs récessifs de restauration qui provoquent des retours à la fertilité ne peuvent être détectés par cette méthode qu'au moment où ils passent à l'état homozygote.

II demeure aussi que l'aptitude au très bon maintien de la stérilité mâle 447 est probablement rare, parce que très polygénique. En partant, comme nous l'avons fait, d'une population qui n'est constituée que de génomes mainteneurs et en appliquant la pression de sélection sur la base du critère ELISA, nous avons retenu après un $\mathrm{BC}_{4}, 15 \%$ de lignées considérées comme très bonnes mainteneuses. Ce taux est tout à fait élevé en comparaison des résultats antérieurs obtenus par notre laboratoire $11 \%$ de lignées bonnes mainteneuses dans une population prise au hasard).

Nos résultats appuyés à présent sur de larges effectifs confirment l'existence d'une liaison entre «teneur en particules» et «stabilité des lignées», qui avait été détectée par Duc et al (1984) sur un faible effectif.

Notre étude présente exploite cette liaison mais aucun de nos résultats ni ceux de la littérature ne permettent de prouver que les particules sont l'agent causal direct de la stérilité mâle «447». Toutefois l'ensemble des propriétés de ce système «447», et notamment le fait que les VLPS soient un marqueur parfait de la restauration permanente et de l'instabilité, favorise ce modèle.

\section{REMERCIEMENTS}

Nous tenons à remercier Madame O Chatagnier qui, par ses compétences et son appui technique, a contribué à ce travail.

\section{RÉFÉRENCES}

Alexander MP (1969) Differential staining of aborted and non aborted pollen. Stain Technol 44, 117-122

Avrameas S (1969) Coupling of enzymes to proteins with glutaraldehydes. Use of the conjugates for the detection of antigen and antibodies. Immunochemistry 6, 43

Bond DA, Fyfe JL, Toynbee-Clarke G (1966) Male sterility in field bean. III. Male sterility with a cytoplasmic type of inheritance. J Agric Sci (Camb) 66, 359-367

Boudon-Padieu E (1989) Detection of Flavescence Dorée-MLO in individual leafhopper vectors during 
latency and inoculative state. Curr Microbiol 19, 357-364

Duc G (1978) Modalités d'expression et hypothèses explicatives du manque de stabilité de la stérilité mâle cytoplasmique de la féverole (Vicia faba $L$ ). Thèse de Docteur-Ingénieur, Univ Paris Sud, $98 \mathrm{p}$

Duc G, Huglo B (1984) Étude du déterminisme paternel de l'instabilité phénotypique de la stérilité mâle. Agronomie 4, 629-637

Duc G, Scalla R, Lefebvre A (1984) New developments in CMS in Vicia faba L. In: Vicia faba agrono$m y$, physiology and breeding (PO Hebblethwaite, TCK Dawkins, MC Heath, G Lockwod-Martinus, DRW Nijhoff, eds) Junk Publishers

Dulieu P, Pénin F, Dulieu H, Gautheron DC (1988) Purification of virus-like particles from male-sterile Vicia faba and detection by ELISA in crude leaf extracts. Plant Sci 56, 9-14

Edwardson JR, Bond DA, Christie RG (1976) Cytoplasmic sterility factors in Vicia faba L. Genetics 82, $443-449$

Letouzey D (1981) Influence de la température sur différents aspects phénotypiques de la stérilité mâle chez la féverole (Vicia faba $L$ ). Thèse de DocteurIngénieur de l'école nat sup agron Rennes
Moussel C (1987) Contribution à la connaissance de la stérilité mâle nucléo-cytoplasmique chez la féverole. Thèse de Docteur de l'Université de Reims, $206 p$

Newman D, Keuls M (1981) Principles and procedures of statistics (RGD Steel, JH Torrie, eds) Mac Grow Hill, Londres

Picard J, Berthelem P, Duc G, Le Guen J (1982) Male sterility in Vicia faba. Future prospects for hybrids cultivars. In: Faba bean improvement (Hawtin G, Webb C, eds) ICARDA Publ

Steef GD, Torrie JH (1960) Principles and procedures of statistics Mc Graw-Hill, 481 p

Thiellement $H$ (1977a) La stérilité mâle chez Vicia faba L. I. Historique et hypothèses. Ann Amélior Plant 27, 203-209

Thiellement H (1977b) La stérilité mâle chez Vicia faba L. II. Hétérogénéité phénotypique d'une lignée mâle-stérile. Ann Amélior Plant 27, 547-553

Thiellement H (1977c) La stérilité mâle cytoplasmique chez Vicia faba L. III. Hérédité des phénotypes polliniques. Ann Amélior Plant 27, 555-562

Thiellement H (1982) Cytoplasmic male sterility in Vicia faba L. VI. Genetical arguments for cytoplasmic heterogeneity. Theor App/ Genet 61, 47-52 